\title{
Neoliberalismo, biocomunicabilidad y la producción de ciudadanos salubres e insalubres. Un análisis de las políticas contra la anemia vistas desde la Amazonía peruana
}

\section{Helen Palma Pinedo}

(iD) https://orcid.org/0000-0001-5329-4880

Universidad Federal de Santa Catarina

helenpalmapinedo@hotmail.com

RESUMEN

A partir de un trabajo etnográfico, se realiza una reflexión crítica sobre los procesos de biocomunicabilidad (Briggs y Nichter, 2009) vinculados con las politicas para combatir la anemia, vistos desde una sociedad multiétnica de la Amazonía peruana con población mayoritariamente awajún y wampis. En ese sentido, se destaca la inserción de estos procesos en una trama de tensiones inter e intraétnicas que los antecede.

El acompañamiento cercano y prolongado de estos procesos permitió identificar aspectos ideológicos y morales, expresados en una visión medicalizante de la anemia, así como la reproducción de un sentido de ciudadanía basado en el rendimiento individualista y funcional a una lógica neoliberal.

Con esto, se espera contribuir al análisis de las políticas oficiales de atención a la población indígena, lo cual se hace relevante en un momento en el que existe una clara demanda del enfoque intercultural y cuando es necesario estar atentos a los posibles procesos de medicalización de la salud indígena.

Palabras clave: biocomunicabilidad, anemia, Amazonía peruana, awajún. 


\section{Neoliberalism, Biocommunicability and the Production of Healthy and Unhealthy Citizens. An Analysis of Policies to combat Anemia seen from the Peruvian Amazon}

\section{ABSTRACT}

Based on an ethnographic study among a multi-ethnic society of the Peruvian Amazon composed primarily of Awajun and Wampis, a critical reflection is made of the biocommunicability processes associated with policies to combat anemia. The analysis highlights the insertion of these processes in a pre-existing web of inter-and intra-ethnic tensions. Careful and prolonged accompaniment of these processes enabled the identification of ideological and moral aspects expressed in a medicalizing vision of anemia, as well as the reproduction of a notion of citizenship based on the productivity, individualism, and functionalism of neoliberal logic. Thus, we hope to contribute to the analysis of official health policies for indigenous populations, relevant at a time when there is a clear demand for an intercultural approach and a necessity to be attentive to possible processes of medicalization of indigenous health.

Keywords: Biocomunicability, anemia, Peruvian Amazon, Awajún. 


\section{INTRODUCCIÓN ${ }^{1}$}

La anemia es definida como la insuficiencia de glóbulos rojos en la sangre, lo que causa una reducción en la capacidad de suministrar oxígeno al organismo para su correcto funcionamiento; siendo la falta de hierro la causa principal identificada, sin descartar otras como la deficiencia de vitaminas y minerales, infecciones parasitarias, enfermedades hereditarias, entre otras ${ }^{2}$. Para 2019, su prevalencia en niños menores de tres años en el Perú fue de 40,1\%, siendo las zonas rurales las más afectadas llegando a niveles que alcanzaban el 60\% (Instituto Nacional de Estadística e Informática [INEI], 2019), lo que nos habla de una distribución desigual de dicha condición. Sin embargo, como menciona Fassin, no basta con que una patología exista para que constituya un problema de salud, sino que hace falta primero que la movilización de agentes le otorgue una existencia social (Fassin, 2004, p. 302).

Tenemos así que, hoy en día, la anemia es una prioridad indiscutible en la agenda de la salud pública peruana. Pocas veces hemos sido testigos en los últimos años de una movilización política y social de la magnitud como la que se viene desenvolviendo alrededor de este tema y a una escala nacional, logrando vincular a actores que traspasan las fronteras de la comunidad salubrista. Sin embargo, la anemia no es en absoluto un problema nuevo en el país ni tampoco las estrategias para combatirla, ya que las primeras intervenciones vinculadas datan de hace más

\footnotetext{
$1 \quad$ El presente artículo muestra un análisis inicial a partir de la información recopilada en el trabajo de campo para mi tesis doctoral en Antropología Social en la Universidad Federal de Santa Catarina. Agradezco la beca recibida de la Coordenação de Aperfeiçoamento de Pessoal de Nivel Superior (CAPES) que hace esto posible, al financiamiento de la Norm and Sibby Whitten Research Fund que facilitó la fase inicial de esta pesquisa, y al INCT Brasil Plural por todo el apoyo financiero y logístico brindado.

2 OMS, https://www.who.int/vmnis/indicators/haemoglobin_es.pdf, consulta realizada el 10/07/19.
} 
de veinte años, como se verá más adelante. ¿Por qué, entonces, y cómo, es que este tema logró posicionarse en los años recientes y bajo qué términos?

En este artículo, propongo que los procesos de biocomunicabilidad (Briggs y Nichter, 2009) desarrollados en el marco de las políticas de salud y de inclusión social han jugado un rol fundamental no solo para el asentamiento del conocimiento vinculado con la anemia, sino también para lo que sería la naturalización de una lógica neoliberal que parte de distintos operadores del Estado vinculados y que se centra en dos aspectos. El primero de ellos es una visión medicalizante de la anemia que lleva a centrar su disminución en el consumo de los artefactos tecnológicos fabricados para ello (en este caso representados por los multimicronutrientes y el sulfato ferroso), conllevando así a una individualización del tratamiento, en detrimento de la atención a las condiciones de vida y hábitat que estos padecimientos ameritan. El segundo aspecto es la reproducción de un sentido de ciudadanía basado en el rendimiento, para el cual el control sobre los cuerpos se hace necesario con el fin de asegurar esa productividad que el sistema neoliberal demanda. Todo esto en el marco de una noción de bienestar global (Fassin, 2004) que deja poco espacio para la diversidad y manifestaciones del buen vivir.

La biocomunicabilidad hace referencia al proceso de producción, circulación y recepción de conocimiento vinculado a la salud, tomando en cuenta cómo aquello que se comunica —y las ideologías que están detrás - afecta a las audiencias y las ubica social y políticamente (Briggs, 2007). Charles Briggs, quien ha realizado contribuciones importantes en el tema, desarrolla esta propuesta a fin de dejar de ver los procesos comunicacionales vinculados con la salud como procesos mecánicos que hay que identificar y analizar. Más que eso, el interés es observar cómo estos pueden ser un tipo de gobernabilidad que crea y jerarquiza formas de subjetividad, así como roles sociales. Yendo más allá, como el autor afirma, cómo se crean, reproducen y naturalizan ideologías y prácticas neoliberales. Por tanto, lo que se propone a partir de esto es una reflexión alrededor no simplemente de prácticas comunicacionales en salud, sino de observar cómo estas se proyectan ideológicamente.

Este marco $^{3}$ ha servido para analizar los procesos de producción de conocimiento sobre varias epidemias. Ejemplo de ello son los trabajos de Briggs (2005) sobre la epidemia de cólera en Venezuela a inicios de los 90 en el que esta llegó a

Vale mencionar que, si bien Briggs propone el término biocomunicabilidad, su uso por otros antropólogos es reducido. Otros autores que trabajan el mismo fenómeno, cómo los citados, utilizan términos como «comunicación biopolítica» o «procesos comunicativos en salud». 
ser imputada a la "cultura indígena», también está el trabajo de Briggs y Nichter (2009), quienes a partir de la epidemia de la gripe H1N1 en EE. UU. analizaron los elementos que intervinieron para que esta se produzca como un problema de salud pública. Además, se encuentra el valioso aporte de Nations y Monte (1996) sobre la epidemia del cólera en el noreste de Brasil, en el que encontraron cómo la campaña comunicacional construida a partir de la metáfora de la guerra acabó siendo percibida por los residentes de las favelas (a la luz de procesos dramáticos ocurridos en ese contexto) como si la guerra fuese contra ellos y no contra el cólera. Por otro lado, Menéndez (2010) desenvuelve una investigación sobre la llamada "gripe aviar» en México a partir de la información difundida en medios de prensa y revistas especializadas de salud pública, subrayando cómo la información era construida y organizada para reforzar la representación social de la constante e inminente peligrosidad de la enfermedad.

En el caso que forma parte de mi análisis, este es un proceso que ha adquirido una dimensión particularmente importante al tener como premisa de partida que «el desconocimiento en la población general sobre la anemia y sus consecuencias e importancia para el desarrollo infantil, de su futuro y del país, es crítico» (Ministerio de Salud [Minsa], 2017, p. 36), por lo cual se plantearon una serie de intervenciones comunicativas y de educación de la cual hemos sido testigos por diferentes medios.

Considero este análisis pertinente en tanto la literatura producida hasta ahora sobre el tema en el país, en parte conformada por estudios cualitativos realizados por profesionales de la salud y de las ciencias sociales, ha estado más preocupada por identificar y observar los problemas en la adherencia a las tecnologías propuestas, conocer las percepciones o representaciones sobre la anemia en las poblaciones intervenidas (preferentemente indígenas), señalar aspectos culturales que podrían ser «barreras» en la lucha contra la anemia, o profundizar en un análisis causal que dé más pistas de por qué no se reduce la anemia a pesar de la gran cantidad de recursos invertidos. En ese sentido, veo a esta producción como un cuerpo teórico conservador (Bonfil Batalla, 1966), en donde la problematización de las ideologías que subyacen estas intervenciones ha sido poco discutida.

Un tema como el que se propone puede adquirir matices muy interesantes cuando lo observamos desde una sociedad multiétnica de la Amazonía como Santa María de Nieva (provincia de Condorcanqui, región Amazonas, Perú), lugar donde se desenvolvió el trabajo de campo que inspira este artículo. Durante ocho meses continuos de 2019 acompañé distintas actividades de la Red de Salud Condorcanqui, del Ministerio de Desarrollo e Inclusión Social (Midis), del 
municipio y de la agencia de cooperación internacional presente en ese momento, programadas como parte de la llamada «lucha» y «cruzada» contra la anemia. Así, pude observar de cerca sesiones demostrativas de preparación de alimentos con insumos que combaten la anemia, talleres de capacitación sobre el tema a agentes comunitarios de salud (ACS), charlas dirigidas a madres de familia, visitas domiciliarias hechas por el personal de salud, entre otros.

Mi participación, en todos los casos, fue aceptada luego de haber manifestado con transparencia los motivos de mi presencia en el lugar e interés en el tema y, además, luego de haber realizado las coordinaciones formales con las autoridades de cada entidad cuando fue necesario. Debo mencionar que, con el tiempo, logré establecer relaciones de confianza y amistad con varias de las profesionales involucradas, por lo que muchas veces fueron ellas mismas las que me avisaron cuando se daría alguna actividad que consideraban sería de mi interés, siempre con una actitud abierta y curiosa a lo que podría responder cada vez que me solicitaron algún comentario o sugerencia luego de cada evento en el que participé. Esto es algo que agradezco hasta ahora y me hace tener una deuda que espero saldar de alguna forma con los aportes que se desprendan al concluir esta investigación. En ese sentido, el análisis que realizaré aquí representa una primera mirada a esta información, ya que este es un trabajo aún en desarrollo, y sin duda cabrán análisis posteriores.

En una primera parte, haré una contextualización que dará cuenta tanto de las principales estrategias e intervenciones desplegadas alrededor de la anemia, así como de Santa María de Nieva. Después, presentaré evidencia que será discutida a la luz de los conceptos y nociones que ya han sido mencionados, para culminar con algunas reflexiones finales.

\section{CONTEXTUALIZACIÓN: DE LAS POLÍTICAS CONTRA LA ANEMIA AL ESPACIO DE INVESTIGACIÓN}

En esta sección me interesa presentar algunos antecedentes políticos e ideológicos que considero relevantes para comprender el telón de fondo en el que se insertan las políticas contra la anemia que forman parte de este análisis. Engarzaré esto con algunas características de mi ámbito de estudio, destacando, sobre todo, algunos aspectos de la cotidianeidad que permitan tener una visión de algunos de los matices de la relación entre el Estado e indígenas y no indígenas presentes. Esto, a fin de no presentar los procesos comunicativos como hechos aislados, sino como parte de una trama de relaciones que están ahí y los anteceden. 
Con relación a estas políticas, como ya fue señalado antes, la anemia no es un problema de salud pública nuevo y, en ese sentido, las medidas para combatirla tampoco. Así, a inicios de 1990, en un escenario en el que el Perú se aplicaba el ajuste estructural (conocido como «fujishock»), una firme liberalización económica, la privatización de empresas, y demás medidas que seguían de manera disciplinada las pautas del llamado Consenso de Washington que llevó a la instauración de una política neoliberal de las más drásticas en la región, se dieron las primeras intervenciones desarrolladas desde el Programa de Crecimiento y Desarrollo (CRED), dirigido a monitorear la salud de los menores de cinco años. En 1993, durante la reforma del sector Salud, se crearon el Proyecto 2000 y el Proyecto de Salud y Nutrición Básica, que colocaban a la nutrición como un área importante de la atención integral de salud, lo que era afín con las medidas de reducción de la pobreza en los llamados países en desarrollo.

Con estos proyectos se impulsaba el involucramiento de miembros de la comunidad como promotores de salud y líderes comunales en la vigilancia del estado de salud y nutrición de los niños en coordinación con los servicios de atención. Cuatro años después, en 1997, se creó el Programa Nacional de Prevención de Deficiencias de Micronutrientes con la intención de afrontar de manera más integral este problema. En 1998, dentro de los Lineamientos de la Política en Salud 1995-2000, se estableció el Plan Nacional de Alimentación y Nutrición 1998-2000 para disminuir la anemia nutricional y desnutrición crónica, el cual ofrecía un paquete de intervención básico que combinaba la alimentación complementaria, educación en salud y nutrición, control de enfermedades prevalentes de la infancia, planificación familiar y salud reproductiva e inmunizaciones.

En el año 2000, el Perú se adhiere a los Objetivos de Desarrollo del Milenio 1990-2015 propuesto por la ONU, comprometiéndose a reducir para el año 2015 la desnutrición crónica del $25 \%$ al $15 \%$, y la anemia en menores de tres años y en mujeres gestantes (de $68 \%$ y $50 \%$ respectivamente) a menos del $20 \%$ en ambos grupos. Esto, como sabemos, no se cumplió. En 2004, se creó la Estrategia Sanitaria Nacional de Alimentación y Nutrición Saludable, pero es en el año 2007 cuando el Estado emprende con mayor fuerza el desarrollo de medidas concretas dirigidas a unir esfuerzos intersectoriales creando el Programa Articulado Nutricional y la Estrategia Nacional CRECER con el objetivo de disminuir la desnutrición y anemia.

Es también en 2007, en un escenario posdesastre luego del terremoto en el sur del país, que se empiezan a distribuir por primera vez los sobres de multimicronutrientes en las zonas afectadas, con la intervención de Unicef, los cuales tenían el 
nombre de «Sprinkles» y eran fabricados por grandes compañías internacionales. Posteriormente, entre los años 2009 y 2010, se decidió desarrollar un piloto para su consumo en tres de las regiones más pobres del país ubicadas en los Andes: Ayacucho, Huancavelica y Apurímac. Su distribución fue ampliándose de forma progresiva a otras regiones, tomando en cuenta los niveles de prevalencia. En este ínterin el producto fue asumiendo algunas adaptaciones al contexto peruano. Una de ellas fue el nombre, que cambió primero a «Estrellitas» y luego a «Chispitas». Con el tiempo, la reducción de la anemia a partir de la suplementación de multimicronutrientes se convirtió en la estrategia principal, lo que motivó el desarrollo de diversos estudios sobre los retos en su adherencia, comprobándose en varios de ellos que esta era baja y presentaba varias dificultades. Uno identificó, por ejemplo, que era de tan solo 24,4\% (Munares-García y Gómez-Guizado, 2016). Aun así, se continuó insistiendo en esta intervención.

En conclusión, tenemos una serie de acciones que fueron ajustándose e intensificándose en el tiempo conforme el país se fue sumando a compromisos internacionales y a una agenda sanitaria global que empezaba a colocar a la anemia como uno de los problemas más importantes, al afectar a alrededor del 25\% de la población mundial ${ }^{4}$. Lo que sí es más reciente es la movilización política y social que vimos en el país en relación con el tema, que en cierta forma se desprende de esta mayor visibilidad de la anemia, pero también de un reclamo ciudadano por la inaceptabilidad de unas cifras que reflejaron un crecimiento económico sostenido por varios años que fue ejemplo para otros gobiernos de la región, pero que no se tradujeron en una mejor salud o alimentación de los niños, lo que tuvo costos políticos para anteriores presidentes y autoridades.

Por otro lado, en los últimos quince años, el país comienza a adoptar con más fuerza políticas que comenzaban a alinearse con las ideas del denominado «pos Consenso de Washington» que reclamaba una mayor presencia del Estado a partir de, entre otras cosas, reformas centradas en lo social. Así, desde 2005 se empieza a ejecutar el programa social de transferencia condicionada de dinero Juntos, tal como ya se había empezado a hacer en otros países. Afín con este nuevo modelo, se suma el protagonismo del Midis, creado en 2011, desde el cual se gestionan los diversos programas sociales, y que comenzó poco a poco a asumir y desplegar diversas acciones contra la anemia; partiendo del hecho de que todos los beneficiarios de los programas (particularmente los niños hasta los

4 OMS, https://www.who.int/vmnis/database/anaemia/anaemia_data_status_t2/es/Consulta realizada el 12/07/19. 
tres años y las mujeres gestantes) deberían ser tamizados y seguir los controles estipulados con el fin de poder acceder a ellos.

Hoy en día, las principales acciones desplegadas desde el Minsa y Midis en alianza con otras entidades involucradas, combinan el tamizaje periódico de hemoglobina, la provisión de multimicronutrientes y sulfato ferroso, las visitas domiciliarias para el monitoreo y consejería, y la promoción de alimentación saludable a partir del consumo de alimentos ricos en hierro, lo que se ha acompañado con campañas comunicacionales de diversa índole.

No pretendo extenderme más en la presentación de estas políticas, pero me interesa dejar claro en este punto que el escenario que describiré en las siguientes páginas, es un reflejo de este interés y preocupación global en el tema que se ha replicado en el país, adquiriendo una dimensión y protagonismo particular en la agenda de salud pública peruana; y luego en las distintas regiones, siendo las poblaciones indígenas de especial atención por su reconocida vulnerabilidad y ser parte importante de la población foco de esta inclusión discursiva promovida desde el Estado.

¿Qué puede emerger de la aplicación de políticas como las descritas en una sociedad multiétnica de la Amazonía? Comenzaré a responder a ello luego de hacer algunas anotaciones sobre el lugar de donde se desprende este análisis. Mi etnografía transcurrió en el distrito de Nieva, específicamente en Santa María de Nieva, capital de dicho distrito. Esta zona, al igual que gran parte de la Amazonía, presenta una larga historia de contactos e intercambios forzados con distintos grupos, así como haber sido foco de distintos programas de colonización, con una destacada presencia religiosa a partir del Instituto Lingüístico de Verano (ILV) y la misión jesuita. En la actualidad, esta zona continúa siendo foco de acciones neocoloniales y fuerzas extractivistas que han perdurado en el tiempo y que se materializan, por ejemplo, en el extenso recorrido que hace por su territorio el oleoducto Norperuano, desde el cual se extrae y traslada petróleo, y que ha presentado múltiples roturas en los últimos años con graves consecuencias en el medio ambiente y la salud. Además de ello, actividades como la minería y la tala ilegal, así como el cultivo y procesamiento de hoja de coca, son un flagelo contemporáneo que han ido involucrando cada vez más a mano de obra indígena, trayendo la muerte de aquellos dirigentes y personas que en algún momento lanzaron su voz de protesta.

Desde la jerga estatal, Santa María de Nieva es clasificada como urbanomarginal, es decir, estamos hablando de un pequeño centro poblado de aproximadamente 3000 habitantes que, como gran parte de la Amazonía, presenta 
una provisión de servicios básicos bastante deficiente. Durante el tiempo que viví allí tuve que adaptarme rápidamente a resolver mis propias cuestiones domésticas con las constantes ausencias de fluido eléctrico, al agua turbia que llegaba por un par de horas al día por las tuberías y que muchas veces era inutilizable, las frecuentes pérdidas de señal de telefonía, etcétera. Es importante anotar esto dado que, como reconoció la directora del Programa Mundial de Alimentos en la reunión más importante de ejecutivos del Perú (CADE-2018), el 50\% de los casos de anemia estaría vinculado con el acceso a agua segura. Lo que se tiene en Nieva es un servicio totalmente precario, donde no existe un sistema de tratamiento del agua y donde los monitoreos realizados arrojan la presencia de bacterias fecales que hacen que la fiebre tifoidea esté fuertemente incorporada hace varios años en la epidemiología local, de la cual (al igual que muchos de sus pobladores) tampoco pude escapar.

Otra característica es que es el lugar alberga las oficinas de todas las instituciones estatales presentes en la provincia, lo que considero hace que se perciba para los que somos de fuera una presencia importante del Estado en un sentido simbólico: las numerosas locaciones que ocupan sus principales vías, la gran cantidad de personal local y foráneo que emplean las distintas entidades que desde muy temprano se ven uniformados recorrer las calles presurosos yendo a trabajar, las numerosas ceremonias y actos solemnes que se desenvuelven todas las semanas por distintos motivos, así como las actividades propias de cada sector, siendo el de salud uno de los más visibles. Santa María de Nieva es, además, un centro de comercio importante para la población de las comunidades aledañas y un lugar de tránsito obligado para aquellos que circulan entre estas y lugares como Bagua, Jaén o Chiclayo. Por ello, una imagen muy presente es la fluidez de sus pobladores y la presencia pasajera de visitantes que llegan y parten, ya sea por la vía fluvial o terrestre.

Cuando se habla de este lugar, se suele destacar la presencia de la población awajún y wampis, obscureciendo peligrosamente la de la población mestiza o apách $^{5} \mathrm{y}$ cruzada ${ }^{6}$. Esta es una dimensión muy importante vinculada con este

5 Categorías étnicas utilizadas en la actualidad para referirse a la población denominada también como «hispanohablante», es decir, aquellos que no son reconocidos como indígenas, y que tienen como lengua materna al castellano. Destaca aquí la población proveniente de Cajamarca y también de lugares como Piura o Chiclayo. Vale decir que la categoría apách tiene una historia bastante más compleja y antigua que lo mencionado aquí, pero para los fines de este artículo me interesa destacar su uso cotidiano actual.

6 Categoría étnica con la cual se autodefinen aquellas personas que son hijos de un emparejamiento awajún/wampis y mestizo. Es bastante frecuente encontrar esta autodefinición, sin 
contexto y que es motivo de una serie de tensiones cotidianas en distintos niveles, y que veo como expresiones de una relación sociorracial compleja que lleva a valoraciones sobre, por ejemplo, quiénes son más educados, más inteligentes, más limpios, más fuertes, más trabajadores, etcétera, lo cual no es raro escuchar en el día a día. Uno de los aspectos que más llamó mi atención desde el inicio fue la expresa necesidad de autoidentificarse y ser identificado étnicamente: «Tú eres apách», «Yo soy cruzado/a», «Él/ella es awajún», eran expresiones cotidianas. Veo esto como parte de una alteridad radical resultado de la vinculación histórica entre indígenas y no indígenas, que exige posicionar y posicionarse ante ese otro, aportando un contenido a las relaciones contemporáneas. Esta dinámica era trasladada también a las esferas de la salud. En una charla a la que asistí, en el que un joven técnico awajún hablaría sobre la anemia y otros temas a un grupo de madres de familia beneficiarias de un programa social, empezó su exposición presentándose y diciendo: "Yo soy awajún, quería saber cuántas de ustedes son apách y quiénes awajún". Más adelante, me presentó ante el grupo como antropóloga y apách. Esto es algo que experimenté en repetidas ocasiones.

En ese sentido, me interesa destacar que los datos que presentaré emergen de un espacio de convivencia multiétnica en el que los diálogos a partir de la anemia son también una manifestación de la diversidad de nociones de salud, enfermedad, y bienestar que se confluyen y confrontan.

En esta misma línea, es importante alejarnos de la imagen binaria y simplista de trabajadores de salud mestizos, apách o blancos versus pacientes indígenas. Por el contrario, en la actualidad, un grupo importante del personal sanitario, y en general de las diversas entidades presentes en Nieva, son indígenas y cruzados. De hecho, algunos de los puestos importantes tanto en salud como en otros sectores son ocupados por indígenas profesionales y técnicos de distintas áreas. Esto lleva, a mi parecer, a una relación un tanto ambigua con estos engranajes locales del Estado, en donde son los propios indígenas, en compañía o bajo la dirección de los no indígenas, los responsables de la aplicación de las biopolíticas y políticas sociales. Así, varios de los mejores informantes que tuve como parte del aparato estatal dedicado a la anemia eran awajún, quienes, en la ejecución de sus responsabilidades, hacían sus propios esfuerzos para disciplinar a sus paisanos para el cumplimiento de las acciones por las que velaban. Un técnico sanitario awajún responsable del monitoreo de uno de los programas sociales y que debía

embargo, no se cuenta con cifras que den información precisa de la pertenencia étnica de esta zona. 
sensibilizar sobre la anemia me decía un día en tono de queja: «Mis paisanos no entienden, no hacen caso a lo que les decimos, mis indicadores están mal y cuando tengo que ir a las reuniones con los responsables de las otras provincias quedo pésimo». A su vez, el personal indígena participante del Estado era muchas veces objeto de chismes por parte de algunas personas, incluso de su propia red familiar. Por ejemplo, una madre de familia me comentaba sobre una prima de ella (también awajún) que trabajaba para otro programa social «ahora a ella ya ni se le ve, no saluda, trabaja y ya se olvidó de nosotros, así son acá». Esto es un ejemplo de las diferencias y tensiones intraétnicas, muchas veces poco evidenciadas y tomadas en cuenta.

Siguiendo las políticas y normas dictadas desde el nivel nacional, la anemia era una preocupación importante para las autoridades en todo Condorcanqui. Al respecto, la información correspondiente al primer trimestre de 2019 señalaba que el $41 \%$ de niños menores de cinco años de la provincia presentaban desnutrición crónica infantil, y que el 33\% presentaba anemia ${ }^{7}$. Para 2018, Amazonas presentó en total 44,5\% de anemia (Endes, 2018). Prevalencias superiores al 40\% son consideradas en el ámbito internacional como un problema de salud pública severo, siendo la suplementación con hierro de carácter obligatorio en el marco de una estrategia integral de nutrición (OMS, 2012).

Una información interesante a conocer es si existe una distribución diferenciada según la pertenencia étnica, lamentablemente es algo que los sistemas de información en salud aún no registran de forma sistemática. No obstante, un estudio realizado hace poco más de una década con población awajún de esta zona señalaba una prevalencia de desnutrición crónica en niños de $33,4 \%$ y de $76,5 \%$ de anemia, mientras que en mujeres en edad fértil la prevalencia de anemia fue de 50,2\% (Huamán-Espino y Valladares, 2006). Asimismo, en un estudio realizado por la Organización Panamericana de la Salud (OPS) hace algunos años en las provincias de Bagua y Condorcanqui, se tiene que el porcentaje de niños indígenas desnutridos en ambas poblaciones fue superior al $50 \%$, mientras que entre la población mestiza las cifras fueron de 12,2\% en Bagua y de 31,7\% en Condorcanqui (OPS, 2012). Cabe mencionar que hace más de cuarenta años Berlin y Markell (1977) concluyeron la casi ausencia de desnutrición y anemia en esta población, lo que abre una discusión en torno a los impactos de los procesos contemporáneos de cambio económicos, sociales y ambientales en la zona.

Información obtenida de los responsables del Plan Articulado Nutricional, en la Red de Salud Condorcanqui. 
Otro último aspecto que considero importante mencionar es que esta provincia ha sido foco de numerosas intervenciones en materia de salud pública y política social, las cuales se fueron intensificando a partir de los hechos lamentables vinculados al «Baguazo» ${ }^{8}$, lo cual aportó más notoriedad a la zona, derivando en una serie de compromisos para una mayor atención y presencia estatal. A partir de ello, se fueron evidenciando los desafíos y urgencias en materia de salud, entre las que destacó el registro de una epidemia de VIH/sida con prevalencias por encima del nivel nacional, y más altas que las presentadas en otros pueblos indígenas y grupos de riesgo (Unicef, 2016), que suscitó una importante concentración de recursos y agentes del Estado, ONG, entidades sanitarias globales y de la cooperación internacional. Hoy en día, al ser conformada en su mayoría por población indígena y cumplir con los indicadores de pobreza, gran parte de los habitantes de Condorcanqui cumple con el perfil de beneficiarios de los distintos programas sociales: Juntos, Pensión 65, Cuna Más, Qali Warma. Esta participación y tipo de relación que plantea el Estado lleva a dinámicas muy palpables que llaman la atención de los ojos foráneos. Así, por ejemplo, cada vez que estaba programado el pago de Juntos, este se constituía como un evento que involucraba a todo el pueblo. Desde muy temprano se instalaba una feria en la parte central donde se vendían ropa y zapatos, además de carpas con personal del Midis que orientaba a las mujeres en el uso del cajero electrónico. Distintos vendedores que venían de otros lados se ubicaban en sus calles principales, ya de por sí llenas de tiendas que también se abastecían para la ocasión, ofreciendo objetos para el hogar, golosinas y otros alimentos, y por supuesto, estaba también la llegada continua desde el amanecer de los botes con las familias de las comunidades aledañas que, luego de hacer la larga fila para cobrar, procedían a comprar distintos productos y regresar en los mismos botes cargados de ellos.

Es en esta relación intensa y dinámica del Estado con los pobladores de este lugar, que se insertan los procesos de biocomunicabilidad que pasaré a describir.

Con este nombre se recuerda al conflicto suscitado en 2009 en los alrededores de Bagua, que acabó con 33 fallecidos y más de 150 heridos (indígenas, mestizos y policías), debido a una falta de consulta del Estado sobre la aprobación de una serie de decretos referidos a las actividades extractivas en la Amazonía. Ese hecho marcó un hito en el escenario sociopolítico y ambiental, así como en el relacionamiento del Estado con la población awajún, cuya presencia se intensificaría a partir de ese evento. 


\section{UNA MIRADA A LOS PROCESOS DE BIOCOMUNICABILIDAD}

En esta sección presentaré los mensajes clave, así como descripciones de algunos eventos que identifico como parte de los procesos de biocomunicabilidad vinculados con la anemia en los que, en todos los casos, el interés principal era informar o capacitar sobre el tema ya sea a madres de familia en el marco de actividades de promoción de la salud, o a ACS que serían los responsables de ir a las viviendas a sensibilizar a las madres sobre el tema. Acompañaré esto con fragmentos de algunas conversaciones que sostuve y que ayudan a ejemplificar lo que argumento en este texto.

Al respecto, mi interés es aproximarme al Estado y sus políticas de una forma que parta de pensarlo, como señalan Maluf y Andrade (2017, p. 175), como un campo de acciones. Sobre ello, las autoras señalan:

Trabajos clásicos y contemporáneos en torno de la antropología del Estado han partido del cuestionamiento de su existencia como una entidad orgánica, un ente substantivo dado, buscando entenderlo como prácticas, acciones y discursos que envuelven un conjunto heterogéneo y contradictorio de actores o agentes sociales, con diferentes disposiciones morales y subjetivas. Más allá de las normas, textos y documentos, el Estado es también lo que se produce en el plano de lo que hacen sus agentes, que en lugar de ser solo ejecutores de las políticas públicas son también aquellos que, en sus prácticas cotidianas, reinventan la política?

Esto tiene que ver con hacer una reflexión basada en lo que Fonseca, Scalco y Castro (2018, p. 274) denominan engranajes cotidianos de poder subyacentes a estas intervenciones destinadas a la regulación de los individuos y poblaciones, así como de control sobre los cuerpos para la obtención de derechos, observando su influencia en la constitución de sujetos contemporáneos y procesos de subjetivación (Fassin, 2009). Al mismo tiempo, no quiero agotar mi discusión al ejercicio de un poder unidireccional, como si fuera algo inerte y estanco, sino que también me interesa destacar las posibles agencias, resistencias, y negociaciones, en un escenario de tensiones no solo interétnicas sino también intraétnicas.

Mi descripción partirá de considerar dos de los mensajes que la biocomunicabilidad sobre la anemia en Nieva tenía fuertemente incorporados, y que vi y escuché manifestarse en diversos espacios y momentos. El primero de ellos tenía que ver con presentar a la anemia como una enfermedad «silenciosa y peligrosa»,

\footnotetext{
$9 \quad$ Traducción propia.
} 
mientras que el segundo consistía en explicitar las consecuencias que tendría a nivel del rendimiento, inteligencia y productividad de las personas.

Con relación al primer mensaje, fue algo que se hizo muy evidente desde la primera sesión de capacitación a ACS que presencié. Como parte de la alianza entre una agencia de cooperación internacional, la Red de Salud Condorcanqui y el Municipio, se realizaron una serie de sesiones de capacitación sobre la anemia (sus causas, síntomas, consecuencias) a ACS mujeres y varones, compuestos por representantes de los barrios y comunidades en las cuales se focalizaría el trabajo. El objetivo final era que, luego de este proceso, realizaran visitas domiciliarias para la aplicación de una encuesta sobre el tema, además de monitorear el cumplimiento de las acciones recomendadas y dar consejería. Los y las ACS, que en su mayoría eran awajún y solo una minoría mestizos, estaban divididos en dos grupos, por lo que se trabajó con ellos en fechas distintas: por un lado, estaban aquellos que correspondían a los barrios de Santa María de Nieva, quienes en todos los casos eran bilingües; por otro, estaban aquellos que habían llegado de las distintas comunidades donde se intervendría, quienes una buena parte o eran monolingües o tenían un dominio limitado del español.

El personal encargado de dirigir las sesiones era identificado como mestizo, por lo que toda la información fue dada en español. Cuando lo consideraron necesario (sobre todo con los ACS de las comunidades) se pidió apoyo a alguno de los asistentes para traducir los mensajes al awajún. Dadas estas características, es posible también observar estos espacios como instancias de traducción e interpretación mutua (Kelly, 2011), que ejemplifican la relación entre el Estado e indígenas y no indígenas.

Durante la primera sesión, luego que se diera información inicial sobre el tema, la enfermera que en ese momento era responsable de la charla comentó: «Todos debemos considerar a la anemia como una enfermedad, y decimos que es una enfermedad silenciosa porque [el paciente] no tiene fiebre, pero lo está matando por dentro». Esta es una idea que fue reforzada y repetida a lo largo de las sesiones. La intención era doble. Por un lado, dejar en claro que la anemia es una enfermedad y, por otro, establecer su peligrosidad. Si bien ya desde el Plan Nacional vigente para el tema (Minsa, 2017) se asume una falta de conocimiento (como fue presentado al inicio de este texto), existía información previa específica de la zona que expresaba ello según un trabajo realizado (Mayca-Pérez, Medina-Ibañez, Velásquez-Hurtado y Llanos-Zavalaga, 2017), lo que reforzaba la necesidad de incidir en este aspecto. 
De esta manera, la premisa de un desconocimiento de la población era el punto de partida (y a la vez preocupación inicial) que guiaba las estrategias comunicativas, que llevaban a explicar «de cero» las nociones sobre esta enfermedad. Así, en una de las reuniones previas para la coordinación y diseño de estas sesiones a la que fui invitada, y en la que se debía pensar en material comunicacional sobre el tema, la discusión giró en torno a la preocupación planteada por la representante de la cooperación internacional que financiaba esta actividad: «¿Cómo le decimos a la mamá que la anemia hace daño?». Como vemos, además de incidir en su peligrosidad, se añade el hecho de incluir a la madre como principal receptora de los mensajes.

Esta premisa atravesaba las diversas intervenciones a las que hice seguimiento. Por ejemplo, en otro momento, una joven awajún responsable del monitoreo y acompañamiento de las familias inscritas en Cuna Más me comentó que las capacitaciones que había recibido tenían como tema central a la anemia, y que la orden recibida de sus superiores era: «Como las madres no ven a sus hijos mal nos decían que es necesario incidir en que la anemia es una enfermedad, y en que tienen que ir al médico y tomar el tratamiento».

Considero que la insistencia en estos aspectos está muy vinculada con las dificultades para su detección fuera del laboratorio, así como a las inconsistencias entre lo que mandan los manuales respecto de sus síntomas y lo que se observa en la realidad. Mol (2002), en su trabajo a partir de la ateroesclerosis, describe las diferentes formas en que esa enfermedad era enactuada, siendo dos de ellas a través de la patología y la clínica. Ella menciona que en ese caso era la versión clínica, basada en los síntomas, la que normalmente prevalecía para el diagnóstico. La anemia sería un ejemplo de lo opuesto: el diagnóstico es definido por la patología, es decir (en este caso) por el nivel de hemoglobina, mientras que la dimensión clínica resulta poco relevante.

Hago hincapié en ese punto, porque este detalle abría un gran espacio para la constante interpelación a la información sobre la enfermedad. Así, lejos de ser oyentes y receptores pasivos, en numerosos momentos se generaron airadas intervenciones, especialmente de las ACS mujeres, quienes, desde su experiencia como madres y abuelas, se manifestaban con expresiones como: «Me dijeron que mi hija tiene anemia, tiene 10,5 de hemoglobina, pero ella corre, juega, no está pálida ni se duerme como ustedes dicen, quiero saber por qué es»o «Mi bebé de cinco meses tiene anemia, pero yo no he tenido en el embarazo, a qué se debe, entonces». Las respuestas iban, por lo general, en la línea de buscar alguna explicación en alguna supuesta falta en los cuidados dados a los niños como: 
«Seguro que tu niña come mucho azúcar o carbohidratos y por eso está bien despierta» $\mathrm{o}$ «Seguro no le has dado bien de lactar y por eso ya tiene anemia». Las expresiones en los rostros con las que quedaban estas mujeres hacían notar su incredulidad en los argumentos ofrecidos. Estos múltiples cuestionamientos que escuché tanto de las ACS awajún como mestizas, mujeres ya habituadas en su mayoría a las charlas y capacitaciones de salud por el rol que desempeñaban, parecía anticipar las inquietudes que sus propias vecinas y paisanas podrían hacerles a ellas cuando tuviesen que visitar sus casas.

En otro momento, dentro de esa misma sesión, se planteó una dinámica. Se repartieron tarjetas entre los asistentes para que cada uno escribiera una causa y una consecuencia de la anemia y luego pegarlas en una pizarra. La dinámica tenía la intención de medir qué tanto los ACS estaban comprendiendo la información que ya había sido dada hasta ese momento. Pasados unos minutos, los fueron llamando por grupos para que ubicaran sus respuestas en el lugar que correspondía. Grande fue mi sorpresa al observar que una de las tarjetas colocada como parte de las causas. Decía: «La anemia no es una enfermedad, es falta de alimentos». Luego, observé otra decir: «La anemia es mal de alimentación, lo que falta es dinero». Pude identificar que quienes las colocaron fueron dos varones awajún, uno joven y otro más mayor.

Acabada esa parte, la enfermera facilitadora procedió a dar una lectura rápida de las tarjetas. Al leer dichos mensajes fueron tomados con algo de gracia, prácticamente no se les dio importancia y se pasó a un siguiente punto. Yo, en mi asiento y con mi visión de antropóloga, no pude evitar gesticular pensando en cómo podían dejar algo así de lado, y no intentar por lo menos averiguar someramente por qué habían escrito eso. Decidí en ese momento no intervenir: no era mi rol, y opté por reservar mis comentarios para el final, que era cuando comúnmente podía conversar con el personal de salud con el fin de dar alguna impresión de la actividad que pudiese servir para alguna mejora posterior. Lo que también hice fue, acabada esa dinámica, acercarme al joven que escribió una de las tarjetas con la intención de tener alguna pista que diera más luces de esa categórica afirmación. Concluyo que probablemente fue un abordaje algo torpe o inoportuno, dado que el joven me miró con un poco de sorpresa, luego negó haberlo escrito y señaló que había sido otra persona, y finalmente sonrió como mostrando su incomodidad sin decir más, a lo que obviamente opté por no insistir y dejar la situación ahí.

Lo que percibí como una potente interpelación a lo que se decía sobre la anemia quedó resumido para mí de manera muy contundente y gráfica en otro 
momento. Esta vez me encontraba de observadora en otra sesión de capacitación en la que participaba un grupo más reducido de las ACS más activas que también estuvieron presentes en las sesiones descritas. Esta vez el objetivo era prepararlas para la aplicación de otro cuestionario para las familias de ciertos barrios seleccionados, que también incluía preguntas vinculadas con la anemia. Luego de aproximadamente hora y media de iniciada la sesión, en la que nuevamente se había incidido en sus causas y consecuencias, el obstetra mestizo encargado de la charla abrió una ronda de preguntas. En ese momento, una de las ACS con más años de experiencia, también mestiza y que vivía hace bastantes años en la zona, levantó la mano para decir: «Licenciado, yo quisiera sufrir la anemia, para sentir qué es». Sus compañeras sonrieron y murmuraron entre ellas respaldando el comentario, en tanto el obstetra que ya conocía a la señora hace bastante tiempo, atinó también a sonreír, no hacer comentario, y continuar con las otras preguntas.

Considero que más allá de discutir en torno a lo insatisfactorio e insuficiente que puede resultar el conocimiento científico sobre la anemia, esto nos muestra un escenario de pugnas y resistencias en lo concerniente a la salud y la enfermedad, al lugar que ocupa el cuerpo en ello, el papel de las condiciones de vida, y lo que debe o no tratarse como un problema a ese nivel. Fassin (2004, p. 297) escribió que la salud no está dada, sino que se produce, y que en ese sentido no siempre se basa en consensos. En ese proceso, muchas veces se optó por medicalizar un problema, para luego politizarlo y convertirlo en un asunto de salud pública demandante de programas e intervenciones sanitarias. Creo que en cierta medida la anemia puede ser una muestra de ello, en donde el énfasis de los aspectos médicos (a pesar de sus posibles inconsistencias) es la justificación para las intervenciones posteriores.

Sin embargo, la biocomunicabilidad sobre este tema no se agota aquí, sino que viene otro punto que observé y que nos da pistas de las nociones de ciudadanía y procesos de subjetivación detrás. Con ello, me refiero a la centralidad otorgada a las consecuencias que la anemia tendría en el rendimiento, inteligencia, productividad, e incluso habilidades sociales de las personas. Durante los días que asistí a esas sesiones de capacitación a ACS, pude registrar múltiples expresiones en esa línea. En todos los casos fueron hechas por el personal de salud responsable de las charlas, cuando explicaban las consecuencias de la anemia en los niños:

Los que hacen anemia desde pequeñitos, de grandes no van a poder trabajar bien $[\ldots]$ Habrá baja productividad, bajos ingresos. 
El niño [con anemia] es inseguro, tiene miedo al fracaso, por ejemplo, en el vóley no sabe si matar a la net. Todo eso es por la falta de estimulación que han tenido [...] Por eso se ven profesionales más tímidos y otros más decididos.

Las consecuencias de la anemia se ven en la escuela, al niño no le gusta [los cursos] Comunicación, Matemática, el profesor llama a los padres porque no participa, no entiende [...] Al niño le preguntan que ha leído, y dice «no sé».

Un niño con anemia no juega, está allí sentado, uno dice que es tranquilo, pero no es que sea tranquilo, está enfermo.

Foto 1. Mural pintado en las afueras de la Red de Salud Condorcanqui.

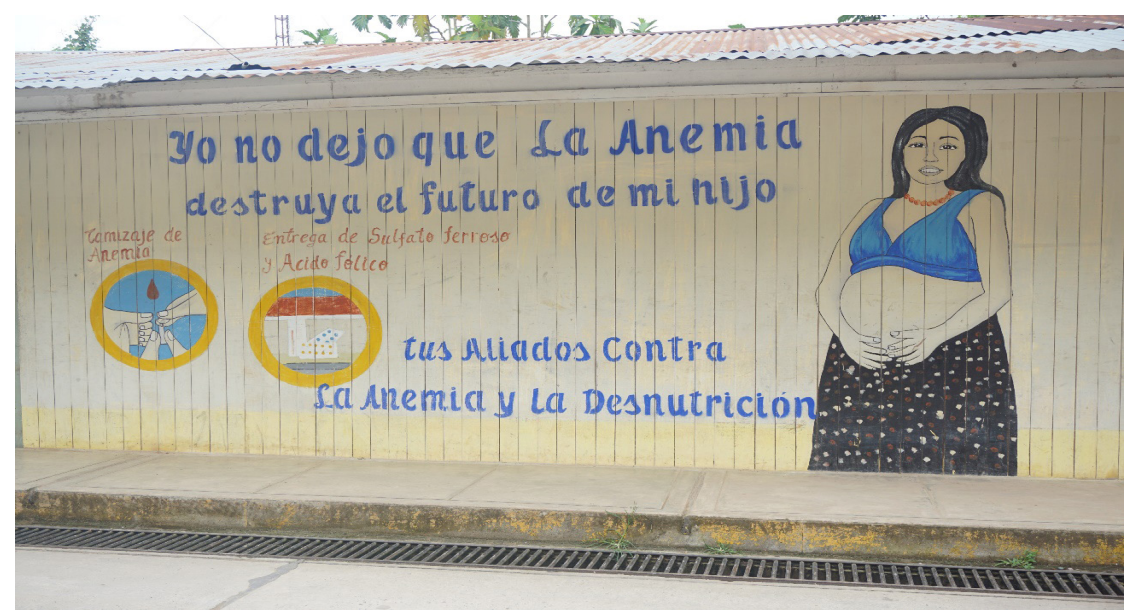

En esa misma línea, durante esa sesión, a pesar de lo poco dialógico y bastante fiel a los manuales que se mostraba la producción del conocimiento sobre la anemia, pude observar algunos intentos de una traducción creativa que invitaba a los asistentes a imaginar los estragos de la anemia en su organismo y capacidades. Para ello, la misma facilitadora presentó de manera entusiasta una cartulina, en la cual estaba dibujada una especie de cuadrícula que señaló era una red de pescar. Luego preguntó. «¿A todos nos gusta pescar no? Y regresar llenos de pescados para comer», a lo que los asistentes respondieron afirmativamente y haciendo algunas bromas. Seguidamente, explicó en tono pedagógico que todos tenemos en nuestra cabeza una red como esa y, por tanto, lo que la anemia hace es agujerearla, lo que trae como consecuencia que los peces del conocimiento 
escapen de nuestro cerebro. Concluyó reforzando el mensaje que nuestras neuronas son como esa red, y que si tenemos anemia nuestra capacidad de retener la información y de ser inteligentes será muy limitada. Las reacciones en este caso expresaron curiosidad y no se presentaron objeciones.

Otro ejemplo lo presencié durante una de mis primeras semanas allí, cuando se dio un acto ceremonial grande como parte del inicio del año escolar en la zona central del pueblo, en donde habían colocado módulos informativos tanto del Midis como del Minsa. En ambos se presentaban platillos preparados con los alimentos identificados como ricos en hierro, además de material informativo sobre la anemia. Como parte de las palabras iniciales de las principales autoridades, se destacó la importancia de combatirla a fin de tener niños sanos, estudiosos, y (como uno destacó) «ciudadanos útiles para la sociedad».

Aunque se podría pensar que este tipo de mensajes forman parte de una producción local de información sobre el tema, es preciso mencionar que el Plan Nacional vigente (Minsa, 2017) contiene un componente especial dedicado a desarrollar una estrategia comunicativa dirigida al cambio de comportamiento en el cual se menciona, por ejemplo, que uno de los ejes temáticos que debe formar parte de los mensajes es «el éxito personal como producto de una buena alimentación en la infancia» (p. 60), y se tienen spots radiales con títulos como «La vida te premia cuando creces sin anemia» (p. 58). Por tanto, lo descrito no es algo aislado, sino que se condice con lo señalado en el documento oficial de alcance nacional.

Estos discursos, que reproducen lo que considero una noción de ciudadanía basada en el rendimiento, individualista y funcional al capitalismo global, además de poco abierta a expresiones y expectativas alternativas de bienestar y buen vivir, van de la mano de estudios económicos que fueron elaborados en su momento para sustentar el costo que tendría la anemia al Estado, lo cual justificaba la inversión en las acciones a tomar ${ }^{10}$. Nociones como esta parecían estar bien incorporadas en otro de los agentes responsables de desplegar las acciones contra la anemia en Nieva. Un enfermero awajún del Midis me mencionó en una conversación: «La anemia es un atraso para el país, para el desarrollo, por eso les tenemos que decir que es una enfermedad [...] Por eso nuestros niños de Cuna

10 El Banco Mundial estimaba que el costo de intervenciones como las de los multimicronutrientes ascendía a $0,3 \%$ del producto bruto nacional, en tanto el costo asociado a estas deficiencias ascendería al 5\% (Sharieff, Horton y Zlotkin, 2006). A estos se suma un estudio económico que estimaba que por la anemia se pierde aproximadamente $0,62 \%$ del producto bruto interno del Perú, es decir, alrededor de S/. 2777 millones (Alcázar, 2012). 
Más tienen que marcar una diferencia, porque van a ser más estimulados, más yachay o inteligentes, como decimos».

Considero que estos ejemplos muestran un aspecto ideológico y moral de la biocomunicabilidad sobre la anemia que nos habla de buenos y malos ciudadanos, de ciudadanos salubres e insalubres. Esto iba respaldado por lo que podría ser una peligrosa reducción de la llamada inclusión a una dimensión productiva, medida en términos estadísticos y económicos, tal como señalan Fonseca y otros (2018) en su trabajo sobre programas sociales en Brasil, ignorando la dimensión moral propia de estas intervenciones, roles y posicionamientos que afloran a un nivel microterritorial. Con ello me refiero, en este caso, a las paradojas que emergen de este modelo de inclusión, que pude observar particularmente para el tema de la anemia. Por un lado, como es sabido, con el interés de contribuir a la equidad de género, Juntos (así como otros programas) prioriza que las titulares del hogar sean las mujeres, haciéndolas sus principales interlocutoras para sus diversas acciones. Sin embargo, esta válida y muy necesaria intención inicial terminaba tornándose en un ejercicio de gobernabilidad que recaía sobre ellas, quienes, además de asumir las labores reproductivas del hogar y de cuidado de varios hijos, tenían el deber de asistir a reuniones y charlas repetitivas en donde el mensaje frecuente era que la anemia es por su descuido y por no saber cómo alimentar bien a sus hijos, además de tener que aceptar todos los tamizajes, vacunas y tratamientos exigidos bajo un esquema de relación poco dialogante y de falta de reconocimiento de los saberes locales y de la diversidad presente en escenarios, como lo puede ser Nieva o muchos otros del Perú.

Esta que llamo «la paradoja de la inclusión» la vi ejemplificada con claridad en una de las mujeres awajún con las cuales llegué a tener una relación más cercana. Ella, quien tenía una historia reproductiva y experiencia de la maternidad difícil y llena de complicaciones por los múltiples abandonos que sufrió de sus parejas, me respondió un día, luego que yo le preguntara si no había pensado en inscribirse en Juntos (dadas las grandes dificultades económicas que estaba atravesando y que la mayoría de mujeres de su barrio estaban inscritas): «No quiero, no me gusta que te obligan, te piden hacer muchas cosas y yo no tengo tiempo, todos los días tengo que ver cómo hacer para que mis hijos coman algo [...] Además, todo el tiempo ponen ampollas, vacunas y uno ni sabe qué enfermedades son esas que dicen». Así, la mujer que, desde mi punto de vista, precisaba ser más «incluida», en un ejercicio de agencia, rechazaba esta inclusión y percibida sujeción que el Estado le proponía a cambio de los S/. 200 bimensuales de Juntos. 
Esta dimensión moral de la biocomunicabilidad sobre la anemia se extendía a valoraciones vertidas sobre la comida. Fueron múltiples las veces que escuché decir que los «nativos» (como eran llamados los indígenas) solo comían yuca y plátano y por eso eran desnutridos, y que había que enseñarles a cocinar otras cosas que sí servían contra la anemia y que sí los iban a alimentar. Un día, una de las señoras mestizas dueña de una de las tiendas más grandes y surtidas, me comentaba que ella les había enseñado a comer a los nativos ciertos tipos de hortalizas y lácteos que vendía en su negocio, porque antes comían con muy poca variedad.

Las sesiones demostrativas de preparación de alimentos son otro buen ejemplo. En una de ellas, el personal responsable de esta actividad (masculino) manifestaba su interés por enseñar a cocinar y dar de lactar correctamente a las mujeres asistentes, mujeres que desde niñas debieron aprender a resolver la alimentación en sus hogares y para quienes la lactancia es parte fundamental de su experiencia de maternidad. Además, expresaba que los tubérculos y frutos locales no servían para la anemia, por lo que se proponía como solución el consumo de la «sangrecita» ${ }^{11}$. El detalle es que en Nieva no la vendían, y que es un producto muy ajeno a los repertorios culinarios locales.

Muy por el contrario, lo que pude vivenciar el tiempo que viví allí fue una centralidad de la comida no solo vista desde un aspecto nutricional, sino de la sociabilidad femenina y entre parientes. Esto lo vi expresado en las largas discusiones y debates sobre qué se comería cada vez que se acercaba un evento social, los intercambios de alimentos entre vecinas y familiares, los momentos compartidos en su preparación intercambiando consejos sobre qué nutría más, etcétera, mostrando una comensalidad que incorporaba y mezclaba los distintos productos alimenticios disponibles.

Aunque observé algunos intentos de los responsables de estas estrategias por identificar recursos locales que pudiesen ser útiles contra la anemia, estos eran aún incipientes. Lo mismo ocurría con aquellos discursos que empezaban a anotar la necesidad de abordar factores como la calidad del agua o la seguridad alimentaria, que parecían aún no calar ni hacer eco en las autoridades que podrían intervenir en ello. Al final, primaba una visión medicalizante de la anemia, atribuyéndole una cura física a partir de una tecnología.

Todo ello podría ser revisado de manera productiva si diésemos más atención a las expresiones de resistencia descritas y a un mayor conocimiento del entorno de aplicación de estas políticas.

11 Alimento preparado a partir de la sangre de (generalmente) pollo. 


\section{REFLEXIONES FINALES}

Las reflexiones propuestas representan una aproximación al Estado y la dimensión biopolítica (Foucault, 1999) de las políticas sobre la anemia vistas «desde abajo», es decir, a partir de las prácticas cotidianas en los servicios e instituciones, y los modos en que los llamados «usuarios» o población objetivo interactúan con estas (Abéles, 1992). En cierta forma, considero que este trabajo responde a la efervescencia de políticas e intervenciones en salud que ha tenido Nieva en los últimos años, lugar donde las interacciones (a veces fluidas, a veces tensas) con el Estado y sus agentes forman parte de la convivencia cotidiana. En ese sentido, creo que el análisis presentado va muy de la mano de la historia reciente de la relación Estado - Amazonía, y de esa necesidad de explorar las nuevas gobernabilidades en salud.

Asimismo, los cuestionamientos señalados no pretenden dejar como mensaje un descarte de las políticas pensadas en lograr una atención de la población, sino revisarlas invitando a pensar en lo que sería poder lograr políticas menos medicalizantes y cuya aplicación esté cada vez más alineada con las preocupaciones locales y particularidades de las zonas de intervención, rescatando saberes y prácticas que, en lugar de ser vistos como barreras, puedan ser integrados.

La discusión sobre la anemia trasciende lo médico y técnico, y nos debe llevar a considerar los retos actuales en la reproducción de los sistemas alimentarios que por mucho tiempo parecieron satisfacer las necesidades de la población awajún y wampis originaria de esta provincia. De otro lado, los procesos de modernización y cambio que impone el sistema neoliberal, que incluye una biocomunicabilidad que contribuye a la clasificación de sujetos que pueden y no desarrollar determinadas habilidades intelectuales basadas en un modelo de individuo occidental, no parece incluir una provisión de servicios básicos digna. Esto es algo que debe ser cuestionado.

Por el momento, la idea de un sujeto individualizado a partir de las nuevas formas de gobierno del cuerpo (Maluf, 2018) y el control sobre él para la obtención de derechos (Fassin, 2003) parece ser lo predominante, en detrimento de la atención necesaria a las condiciones de hábitat, seguridad y soberanía alimentaria. Incluir el abordaje de las relaciones de género con relación a los cuidados es otro pendiente. Con ese telón de fondo, la meta de reducir la anemia a 19\% para 2021 parece ser vista como alcanzable teniendo solo como solución primordial a las intervenciones tecnológicas, aunque con ello se corra el riesgo de ahondar las tensiones y brechas en la relación entre el Estado y la población, particularmente las mujeres, dada la escasa aceptación a este tipo de medidas y control. 
Si bien el análisis a partir de la biocomunicabilidad ha dado luz a aspectos poco discutidos, profundizar en las historias de los niños y mujeres foco de estas intervenciones y en la complejidad de los modos de comensalidad es otro pendiente, y motivo de trabajo actual de esta investigación.

\section{REFERENCIAS}

Abélès, Marc (1992). État. En Pierre Bonte y Michel Izard (dirs.), Dictionaire de l'ethnologie et de l'anthropologie (pp. 239-242). París: PUF.

Alcázar, Lorena (2012). Impacto económico de la anemia en el Perú. Lima: GRADE; Acción contra el Hambre.

Berlin, Elois Ann y Edward K. Markell (1977). An Assessment of the Nutricional and Health Status of Aguaruna Jívaro Community, Amazonas, Peru. Ecology of Food and Nutrition, 6(2), 69-81. https://doi.org/10.1080/03670244.1977.9990483

Bonfil Batalla, Guillermo (1966). Conservative Thought in Applied Anthropology: A Critique. Human Organization, 25(2), 89-92. https://doi.org/10.17730/ humo.25.2.b3184896167268r2

Briggs, Charles L. (2005). Perspectivas críticas de salud y hegemonía comunicativa: aperturas progresistas, enlaces letales. Revista de Antropología Social, 14, 101-124. Recuperado de https://revistas.ucm.es/index.php/RASO/article/view/ RASO0505110101A

Briggs, Charles L. (2007). Anthropology, Interviewing, and Communicability in Contemporary Society. Current Anthropology, 48(4), 551-580. https://doi. org $/ 10.1086 / 518300$

Briggs, Charles L. y Mark Nichter (2009). Biocommunicability and the Biopolitics of Pandemic Threats. Medical Anthropology, 28(3), 189-198. https://doi. org $/ 10.1080 / 01459740903070410$

Fassin, Didier (2003). Gobernar por los cuerpos, políticas de reconocimiento hacia los pobres y los inmigrantes en Francia. Cuadernos de Antropología Social, 17, 49-78. https://doi.org/10.34096/cas.i17.4599

Fassin, Didier (2004). Entre las políticas de lo viviente y las políticas de la vida. Hacia una antropología de la salud. Revista Colombiana de Antropología, 40, 283-318.

Fassin, Didier (2009). Another politics of life is possible. Theory, Culture and Society, 26(5), 44-60. https://doi.org/10.1177/0263276409106349

Fonseca, Claudia, Lucia Mury Scalco y Helisa Canfield de Castro (2018). Etnografia de uma política pública: controle social pela mobilização popular. Horizontes. Antropológicos [online], 24(50), 271-303. https://doi.org/10.1590/ s0104-71832018000100010 
Foucault, Michel (1999). Em defesa da sociedade. São Paulo: Martins Fontes.

Huamán-Espino, Lucio y Carmen Valladares (2006). Estado nutricional y características del consumo alimentario de la población. Aguaruna, Amazonas. Perú 2004. Revista Peruana de Medicina Experimental y Salud Pública, 23(1), 12-21.

Kelly, José Antonio (2011) Equívocos sobre identidad y cultura: un comentario sobre la formulación de políticas para los pueblos indígenas en Venezuela. En German Freire (org.), Perspectivas en salud indígena: cosmovisión, enfermedad y políticas públicas (pp. 417-462). Quito: Abya-Yala.

Maluf, Sônia Weidner (2018). Antropologias e políticas em contextos de crise. En Rosana Castro, Cíntia Engel, Raysa Martins (orgs.), Saudades do futuro (pp. 24-35). Brasília: Sobrescrita.

Maluf, Sônia Weidner y Ana Paula Müller de Andrade (2017). Entre políticas públicas e experiências sociais: impactos da pesquisa etnográfica no campo da saúde mental e suas múltiplas devoluções. Saude e Sociedade, 26(1), 171-182 [online]. https://doi.org/10.1590/s0104-12902017168331

Mayca-Pérez, Julio, Armando Medina-Ibañez, José E. Velásquez-Hurtado y Luis F. Llanos-Zavalaga (2017). Representaciones sociales relacionadas a la anemia en niños menores de tres años en comunidades Awajun y Wampis, Perú. Revista Peruana de Medicina Experimental y Salud Pública, 34(3), 414-422. https://doi. org/10.17843/rpmesp.2017.343.2870

Menéndez, Eduardo (2010). Las influenzas por todos tan temidas o de los difíciles usos del conocimiento. Desacatos, 32, 17-34.

Ministerio de Salud (2017). Plan Nacional para la Reducción y Control de la Anemia Materno Infantil y la Desnutrición Crónica Infantil en el Perú: 2017-2021. Lima: Minsa.

Mol, A. (2002). The Body Multiple: Ontology in Medical Practice. Durham, NC: Duke University Press.

Munares-García, Oscar y Guillermo Gómez-Guizado (2016). Adherencia a multimicronutrientes y factores asociados en niños de 6 a 35 meses de sitios centinela. Ministerio de Salud, Perú. Revista Brasileira de Epidemiología, 19(3), 539-553. https://doi.org/10.1590/1980-5497201600030006

Nations, Marilyn K. y Cristina M. G. Monte (1996). «I'm not dog, no!»: Cries of resistance against cholera control campaigns. Social Science \& Medicine, 43(6), 1007-1024. https://doi.org/10.1016/0277-9536(96)00083-4

Organización Panamericana de la Salud (2012). Estado de salud y nutrición de los niños menores de 5 años pertenecientes a las poblaciones indigenas y no indígenas de Bagua y Condorcanqui en la región Amazonas, 2012. Informe técnico. 
Sharieff, Waseem, Susan E. Horton y Stanley Zlotkin (2006). Economic gains of a home fortification program: evaluation of «Sprinkles» from the provider's perspective. Canadian Journal of Public Health, 97(1), 20-23. https://doi.org/10.1007/ BF03405206

Unicef (2016). VIH en población indigena awajún. S.1.: Unicef. 